Ann. Biol. anim. Bioch. Biophys., r976, 16 (6), 89I-895.

NOTE

\title{
EFFET A MOYEN TERME DE LA TENEUR EN MAGNÉSIUM D'UN RÉGIME RICHE EN HUILE DE PRIMOR SUR L'APPARITION DES LESIONS DU MYOCARDE CHEZ LE RAT
}

\author{
A. POINTILLART \\ avec la collaboration technique de Colette Cours \\ Station de Recherches de Nutrition, \\ Centre national de Recherches zootechniques, I. N.R.A., \\ 78350 Jouy en Josas

\section{RÉSUMÉ}

Les rats recevant pendant un mois un régime contenant $\mathbf{I}_{5} \mathrm{p}$. Ioo d'huile de Primor et seulement 60 p.p.m. de magnésium, ont présenté des lésions cardiaques d'infiltration histiocytaires et des calcifications dans différents organes (cœur et reins). Les rats recevant le même régime avec 600 ou 4700 p.p.m. de magnésium n'ont pas présenté ces lésions.

\section{INTRODUC'TION}

L'huile de colza, riche en acide érucique, provoque des lésions du myocarde chez le Rat (Rocouelin et al., 1973). La teneur du régime en magnésium peut influer sur le délai d'apparition ou l'intensité de celles-ci (Pointillart et Meslin, 1975; Pointillart et Françors, i975); par contre, avec les huiles de crucifères sans acide érucique, comme celles de Canbra ou de Primor, certains auteurs observent des lésions (Rocguelin et al., i973; Levillain et al., i974; Kramer et al., I973; BEARE-Rogers et al., 1974) tandis que d'autres n'en observent pas (ABDelLAtIF et Vles, I97o ; Dallochio et al., I973). Dans le but d'étudier les interactions possibles du magnésium alimentaire et des effets éventuels de l'huile de Primor, un essai précédent (PorNTILLART et MESLIN, I975) a été repris en utilisant cette huile à la place de celle de colza. L'essai est basé sur l'étude des modifications anatomopathologiques du myocarde après un mois de régime. 


\section{MATÉRIEL E'T MÉTHODES}

1 Des rats mâles Wistar de zoo g environ, divisés en 3 lots de 8 (PO, PN, PS) et répartis en cage par 2, sont nourris ad libitum pendant 30 jours avec un aliment semi-synthétique contenant I5 p. Ioo en poids sec d'huile de Primor ( $\left.{ }^{\mathbf{1}}\right)$. A cet aliment est incorporé un mélange minéral et ( vitaminique dépourvu de magnésium. Cet élément y est ajouté sous forme de chlorure afin d'obtenir respectivement pour les carencés (PO), les normaux (PN) et les surchargés (PS) 6o, ( 600 et 4700 p.p.m. de $\mathrm{Mg}$ dans la matière sèche consommée, teneurs comparables à celles utilisées dans l'essai avec l'huile de Colza (CO : 70, CN : 600, CS : 4 300 p.p.m. de Mg) (Pointillart et MesLin, 1975). Au bout de 30 jours, les coeurs encore battants sont immédiatement prélevés en cours d'autopsie et fixés dans le formol neutre à Io p. Ioo, après partage transversal en 3 morceaux d'égale épaisseur, dénommés pointe $(\mathrm{P})$, milieu $(\mathrm{M})$ et haut $(\mathrm{H})$ en fonction de leur situation par rapport au grand axe. Chaque cœur a permis de réaliser 3 blocs d'inclusion dans le paraplast et chaque bloc a donné lieu à 2 coupes, soit 6 lames par cour ; une lame par niveau a été colorée au Trichrome de Masson pour visualiser les lésions, l'autre au nitrate de cobalt (méthode de STotlzNer) pour mettre en évidence le calcium. Toutes les lames ont été examinées en microscopie optique, mais seules les lésions apparaissant au binoculaire ont été comptabilisées (grandissement entre $\times$ ro et $\times 40$ ) en taille et en fréquence, l'échelle étant donnée par du papier millimétré. La quantification des lésions d'infiltration et de fibrose ne prend en compte que leur longueur dans la plus grande dimension et non leur surface. Le but de la méthode ainsi utilisée est de permettre la comparaison de la gravité des lésions d'un lot à l'autre et non pas de faire une étude descriptive détaillée des divers types de lésions susceptibles d'apparaître. Par ailleurs, les plasmas, les reins et les fémurs ont été minéralisés afin de déterminer leur contenu en calcium et en magnésium.

\section{RÉSULTATS}

\section{I. - Données générales}

Tous les rats carencés en magnésium ont présenté les symptômes cliniques classiques de la carence, notamment l'hyperémie des extrémités (oreilles, queue, pattes) et des lésions cutanées à la fois sur la tête, l'abdomen, les oreilles, les lèvres, autour des yeux, avec parfois des traces d'épistaxis. L'apparition des symptômes est rapide (dès la fin de la première semaine) la plupart d'entre eux ayant régressé et les lésions ayant cicatrisé à l'abattage. Les croissances des rats sont comparables sauf vers la fin où celles des carencés diminuent significativement. Les carencés ont consommé globalement et en moyenne moins d'aliment ( $12,5 \mathrm{~g} / \mathrm{jour}$ ) que les normaux (I6,7 g/jour) ou les surchargés ( $15,3 \mathrm{~g} /$ jour).

Les poids des cours ne sont pas significativement différents.

\section{2. - Données biochimiques (tabl. $\mathrm{x}$ )}

Les différences significatives qui apparaissent traduisent essentiellement les effets de la teneur en $\mathrm{Mg}$ du régime : hypomagnésémie, accumulation anormale de calcium dans les reins, abaissement du contenu en $\mathrm{Mg}$ du fémur chez les carencés; hypermagnésémie et élévation du $\mathrm{Mg}$ du fémur chez les surchargés.

\section{3. - Données histologiques}

Seuls les rats carencés en magnésium (lots PO) ont présenté des lésions d'infiltrations (tabl. 2) ou de fibrose. Ce sont essentiellement des lésions d'infiltration histiocytaire de longueur variant entre 0,25 et $2 \mathrm{~mm}$, les lésions les plus fréquentes étant de $0,25 \mathrm{~mm}$ environ. Les plus nombreuses

(1) L'huile de Primor nous a été gracieusement fournie par M. MoRdret de l'Institut des Corps Gras (I. T. E. R. G.). 


\section{TABLEAU I}

Effet de la teneur en $M g$ sur le contenu en $\mathrm{Ca}$ et $\mathrm{Mg}$ du plasma, des reins et du fémur

\begin{tabular}{|c|c|c|c|}
\hline $\begin{array}{l}\text { Régimes } \\
\text { p.p.m. de } \mathrm{Mg}\end{array}$ & $\begin{array}{l}\text { PN } \\
600\end{array}$ & $\begin{array}{c}\text { PO } \\
60\end{array}$ & $\begin{array}{l}\text { PS } \\
4700\end{array}$ \\
\hline $\mathrm{Mg} / 100 \mathrm{ml}$ de plasma $\begin{cases}\mathrm{Ca} & \ldots \\
\mathrm{Mg} & \ldots\end{cases}$ & $\begin{array}{c}10,2 a^{*} \\
2,40 a\end{array}$ & $\begin{array}{c}11,9 b \\
1,12 b\end{array}$ & $\begin{array}{r}10,6 a \\
2,80 c\end{array}$ \\
\hline $\mathrm{Mg} / \mathrm{g}$ de rein sec $\quad \begin{cases}\mathrm{Ca} & \ldots \\
\mathrm{Mg} & \ldots\end{cases}$ & $\begin{array}{l}0,80 a \\
1,64 a\end{array}$ & $\begin{array}{l}8,96 b \\
1,66 a\end{array}$ & $\begin{array}{l}0,78 a \\
1,73 a\end{array}$ \\
\hline P. 100 de fémur sec $\begin{cases}\mathrm{Ca} & \ldots \\
\mathrm{Mg} & \ldots\end{cases}$ & $\begin{array}{r}18,3 a \\
3,5 a\end{array}$ & $\begin{array}{r}18,9 a \\
1,8 b\end{array}$ & $\begin{array}{r}19,1 a \\
4,3 c\end{array}$ \\
\hline Poids du cœur frais $(g) \ldots \ldots$. & $0,83 a$ & $0,71 a$ & $0,78 a$ \\
\hline
\end{tabular}

* Les moyennes non suivies d'une même lettre sont significativement différentes pour $\mathrm{P}<0,05$ par le test des blocs.

TABLEAU 2

Lésions d'infiltrations histiocytaires (I)

et dépóts calciques (C) rencontrés dans les cours des rats carencés en Mg (lot $\mathrm{PO}$ ).

Contenu en calcium des reins

\begin{tabular}{|c|c|c|c|c|c|c|c|c|}
\hline \multirow{2}{*}{ Rat } & \multicolumn{2}{|c|}{ Haut } & \multicolumn{2}{|c|}{ Milieu } & \multicolumn{2}{|c|}{ Pointe } & \multirow{2}{*}{ Total } & \multirow{2}{*}{$\begin{array}{l}\text { Ca rénal } \\
\mathrm{mg} / \mathrm{g} \mathrm{sec}\end{array}$} \\
\hline & I & C & I & $\mathrm{C}$ & $I$ & $\mathrm{C}$ & & \\
\hline 9 & $0,4^{*}$ & $+^{* *}$ & 5,2 & + & 1,75 & - & 7,35 & 1,1 \\
\hline 10 & 1,0 & \pm & 3,5 & - & 2,0 & - & 6,5 & 1,7 \\
\hline 11 & - & - & 1,0 & - & - & - & 1,0 & 1,1 \\
\hline 12 & 1,75 & \pm & 12,0 & \pm & 6,1 & ++ & 19,85 & 8,1 \\
\hline 13 & 0,5 & \pm & 1,5 & - & 4,5 & - & 6,5 & 4,2 \\
\hline 14 & 0,75 & 土 & 2,75 & - & 1,0 & - & 4,5 & 1,0 \\
\hline 15 & 3,75 & + & 5,5 & ++ & 4,75 & ++ & 14,0 & 42,7 \\
\hline 16 & - & 一 & 2,25 & - & - & - & 2,25 & 11,8 \\
\hline Total & 8,15 & & 33,7 & & 20,1 & & 61,95 & \\
\hline
\end{tabular}

Total lésionnel calculé en prenant $1 \mathrm{~mm}$ de lésion $=1$.

**+ Un dépôt calcique de plus de $200 \mu$.

++ Deux dépôts calciques de plus de $200 \mu$.

\pm Dépôts granuleux disséminés. 
se trouvent dans les ventricules (blocs $\mathbf{M}$ et $\mathrm{P}$ ) tandis qu'elles sont absentes des oreillettes (blocs $H)$. Les lésions fibreuses $(2,5 \mathrm{~mm}$ pour le total des 8 carencés) sont rares et peu étendues dans cet essai, comparativement aux lésions d'infiltration $(61,9 \mathrm{~mm}$ pour le total des 8 carencés). Des dépôts calciques de taille avoisinant les $250 \mu$ de long ont été rencontrés dans 6 coupes cardiaques sur 24 examinées soit 3 rats sur 8 dans le lot carencé en magnésium seulement (tabl. 2); dans plusieurs cas, ces dépôts sont superposés aux lésions d’infiltration histiocytaires.

\section{DISCUSSION ET CONCLUSION}

Dans le présent essai, le fait que les lésions n'apparaissent qu'avec le régime $P O$ (carencé en magnésium) est à rapprocher du fait constaté dans l'essai sur l'huile de colza (Pointillart et MesLin, I975) où les lésions les plus nombreuses apparaissaient chez les rats soumis au régime CO (également carencé en $\mathrm{Mg}$ ), ce qui nous conduit à penser que les lésions d'infiltrations apparues avec $\mathrm{PO}$ sont le fait essentiel de la carence en $\mathrm{Mg}$. Il faut remarquer qu'avec les régimes contenant de l'huile de colza, quel que soit l'apport de magnésium (normal, insuffisant ou excessif) des lésions d'infiltrations ou de fibrose dans le cœur des rats, apparaissent déjà à 3o jours, ce qui n'est pas le cas avec l'huile de Primor pour un apport normal ou excessif de magnésium. Toutefois, avec les huiles de crucifères pauvres en acide érucique, le délai d'apparition des lésions joue un rôle sans doute important puisque certains auteurs n'en rencontrent pas à i 5 jours (ABDELlatif et VLES, 1970 ; DALlochio et al., r973) et d'autres en rencontrent très peu après I mois (RocQUELIN et al., I973; Kramer et al., r973) ou davantage après 2 mois (Rocouelin et al., I973; Levillain et al., I974) ; après 4 mois, il y a encore des lésions (Levillain et al., I974 ; BEARERogers et al., I974) ; après 6 mois, Levillain et al., 1974 en observent contrairement à ABDELLATIF et VLES (I970).

Notons, par ailleurs, qu'avec l'huile de Primor, la surcharge en magnésium reste sans effet alors qu'elle agissait à 30 jours, avec l'huile de colza, à la fois sur les lésions et sur la croissance. La constation faite chez deux rats carencés, de lésions cardiaques importantes (infiltrations histiocytaires et calcifications) coïncidant avec des calcifications rénales (néphrocalcinose constatée biochimiquement) montre bien que ces lésions sont dues essentiellement à la carence en magnésium et non à l'huile de Primor. Les animaux carencés ont une croissance et une consommation inférieures à celles des surchargés ou des normaux ce qui peut également intervenir dans l'étiologie des lésions ainsi constatées ; cependant, notons que ces animaux, bien qu'ayant consommé par voie de conséquence moins d'huile de Primor que les autres, ont eu davantage de lésions : il faut donc encore voir là un effet de la carence du régime en magnésium. Avec $P O$, peu de lésions fibreuses ont été mises en évidence, du moins avec le grandissement utilisé, celui-ci étant cependant identique à celui de l'essai avec CO (colza, carencé) or, la carence par elle-même (Greenberg et al., I936) peut provoquer ce type de lésion ainsi que les lésions de calcifications (Heggtveit et al., I964); signalons également qu'aussi bien l'huile de colza (Rocguelin et al., I973) que la carence Mg (NAKAMURA et al., I96I) peuvent provoquer des lésions mitochondriales. En conséquence, l'aggravation des lésions remarquées avec le régime $\mathrm{CO}$ est bien due à la fois à la carence $\mathrm{Mg}$ et à l'huile de colza.

En résumé, si l'on compare les effets simultanés de la teneur du régime en magnésium et de la nature de l'huile (colza ou Primor) la carence on magnésium et l'huile de colza provoquent sans doute le même type de lésions cardiaques chez le Rat. Avec l'huile de Primor, les lésions n'apparaissent dans les mêmes délais qu'en cas de carence en $\mathrm{Mg}$; elles ne sont donc pas le fait de l'huile de Primor, mais de la carence elle-même.

Ręu pour publication en avril 1976. 


\section{REMERCIEMENTS}

Nous remercions très sincèrement J.-C. MESL:N pour les conseils qu'il nous a fournis.

\section{SUMMARY}

\section{ROLE OF MAGNESIUM LEVEL IN A DIET CONTAINING PRIMOR OIL. MEDIUM-TERM EFFECT CN CARDIAC LESIONS IN RAT.}

Rats fed during one month with a diet ccntaining 15 p. roo of Primor oil (zero erucic acid rapeseed oil) and only 60 p.p.m. of magnesium present histiocytic infiltration lesions in the heart and soft tissue calcification in the heart and kidneys. When fer the same diet with 600 or 4700 p.p.m. of magnesium, they present no lesions.

\section{RÉFÉRENCES BIBLIOGRAPHIQUES}

Abdellatif A. M. M., Vles R. O., I970. Physiopathological effects of rapeseed-oil and Canbra oils in rats. Proc. Int. Conf. Sci. Technol. Marketing of Rapeseed and Rapeseed Products, Sainte-Adèle, p. 423-434.

Beare-Rogers J. L., Nera E, A., Heggtveit H. A., r974. Myocardial alteration in Rats fed rapeseed oils containing high or low levels of erucic acid. Nutr. Metabol., 17, 213-222.

Dallochio M., Larrue J., Rabaud M., Razaka G., Crockett P., Brigaud H., i973. Modifications du myocarde de Rat sous l'effet de régimes contenant différentes huiles (arachide, canbra, colza). C. R. Soc. Biol., 167, 257-262,

Greenberg D. M., Anderson D. E., Tufts E. V., I936. Pathological changes in the tissues of rats reared on diets low in magnesium. J. Biol. chem., 114.

Heggtveit H. A., Herman L., Mishra R. K., r964. Cardiac necroses and calcification in experimental magnesium deficiency. Amer. J. Path., 45, 757-782.

Kramer J. K. G., Mahadevan S., Hunt J. R., Sauer F. D., Corner A. H., Charlton K. M., 1973. Growth rate, lipid composition, metabolism and myocardial lesions of rats fed rapeseed oils.' J. Nutr., 103, I696-1703.

Levillain R., Cluzan R., Vodovar N., Desnoyers F., Causeret J., Rocquelin G., 1974.

Aspects anatomo-pathologiques du myocarde de rats ingerant de l'huile de Primor. C. R. Soc. Biol., 168, $475-478$.

Nakamura M., Nakatini M., Kolke M., Tori S., Kiramatsu M., I96r. Swelling of heart and liver mitochondria from magnesium deficient rats and its reversal. Proc. Soc. Exp. Bicl. Med., 108, 3I5-3I9.

Pointillart A., Meslin J.-C., I975. Effet à moyen terme de la teneur en magnésium d'un régime riche en huile de colza sur l'apparition des lésions du myocarde chez le Rat. Nutr. Metabol., 19, Io-19.

Pointillart A., François A., I975. Effet à court terme de la tencur en magnésium d'un régime riche en huile de colza sur le contenu en lipides, du cœur et la teneur en minéraux des tissus mous. C. R. Acad. Sc., Paris, Sér. D, 280, 105-108.

Rocouelin G., Sergiel J.-P., Astorg P. O., Cluzan R., r973. Effets des huiles de colza et de canbra sur les lipides et l'anatomie du myocarde du rat : étude à ccurt terme. Ann. Biol. Bioch. Biophys., 13, 587-609.

Rocouelin G., Cluzan R., Vodovar N., Levillain R., IG73. Recherches récentes sur les effets physiopathologiques des huiles de colza et de canbra au niveau du myocarde et d'autres organes. Cah. Nut. Diet., 8, : I06-I 16. 